\title{
Enhanced antitumor effects of BPD-MA-mediated photodynamic therapy combined with adriamycin on breast cancer in mice
}

\author{
Zhong-sheng TONG*, Pei-tian MIAO, Ting-ting LIU, Yong-sheng JIA, Xiao-dong LIU \\ Medical Department of Breast Oncology, Tianjin Medical University Cancer Institute and Hospital, Tianjin 300060, China
}

\begin{abstract}
Aim: Photodynamic therapy (PDT) is an emerging treatment used to eradicate premalignant and early-stage cancers and to reduce tumor size in end-stage cancers. In this study, we investigated the effects of a combination of benzoporphyrin derivative monoacid ring A (BPD-MA)-mediated PDT with adriamycin (ADM) on 4T1 breast carcinoma cells in vivo and the mechanisms underlying this effect. Methods: Normal BALA/c female mice bearing 4T1 breast carcinoma xenografts were tested. The animals were treated with PDT (BPD-MA $1 \mathrm{mg} / \mathrm{kg}$, iv, plus single-dose laser irradiation) or ADM (5 mg/kg, iv) alone, or a combination of PDT with ADM. The tumor growth rate was determined by measuring the tumor weight. Cell apoptosis was measured with flow cytometry, and the expression of apoptosis-related molecules was assessed using Western blot. Microvessel density (MVD) was determined with immunohistochemical staining.

Results: Compared to PDT or ADM alone, PDT plus ADM produced a combined inhibition on the tumor growth, prolonged life span, and enhanced apoptosis in the mice bearing 4T1 subcutaneously xenografted tumors. The combination of PDT and ADM exerted additive effects on the upregulation of Bax and the downregulation of $\mathrm{Bcl}-2$, and on the reduction of MVD in 4T1 xenografted tumors. Conclusion: Our results demonstrate that PDT plus ADM exerts enhanced in vivo antitumor effect on breast cancer, which is closely associated with the cooperative regulation of extrinsic apoptotic pathways and the inhibition of tumor angiogenesis. Thus, PDT plus $\mathrm{ADM}$ is a promising combined treatment strategy for breast carcinoma.
\end{abstract}

Keywords: breast cancer; photodynamic therapy; benzoporphyrin derivative monoacid ring A (BPD-MA); adriamycin; xenograft; apoptosis; Bax; Bcl-2; angiogenesis

Acta Pharmacologica Sinica (2012) 33: 1319-1324; doi: 10.1038/aps.2012.45; published online 30 Jul 2012

\section{Introduction}

Breast cancer is the most common malignant disease among women in China. For management purposes, breast cancer is categorized into operable or advanced breast cancer. Advanced breast cancer is either a locally advanced or metastatic disease. Locally advanced breast cancer (LABC) is characterized by varying clinical presentations, such as the presence of a large primary tumor $(>5 \mathrm{~cm})$, associated with or without skin or chest-wall involvement or with fixed (matted) axillary lymph nodes or with disease spread to the ipsilateral internal mammary or supraclavicular nodes in the absence of any evidence of distant metastases ${ }^{[1,2]}$. This wide presentation spectrum makes the management of LABC a challenge for oncologists.

\footnotetext{
* To whom correspondence should be addressed.

E-mail tonghang@medmail.com.cn

Received 2012-01-06 Accepted 2012-04-05
}

Photodynamic therapy (PDT) is increasingly being recognized as an attractive alternative treatment modality for superficial cancer ${ }^{[3,4]}$, and this therapy has already been used on patients suffering from cancers of the skin or mouth. Additionally, it is presumed that this form of therapy may replace the use of radiotherapy in a number of breast cancer patients. Wyss et al showed that PDT offers a minimally invasive outpatient treatment modality for recurrent breast cancer on the chest wall with few side effects, high patient satisfaction and possible repetitive application ${ }^{[5]}$.

PDT utilizes a photosensitizer and light to generate reactive oxygen species and creates an opportunity for targeted lesion destruction. Careful selection of the photosensitizer and light source is required to achieve the desired efficacy during the PDT treatment of cancer. Benzoporphyrin derivative monoacid ring A (BPD-MA) is a second-generation photosensitizer for PDT that has yielded good results in phase I clinical trials $^{[6]}$. However, BPD-MA-mediated PDT in breast carcinoma is still obscure. 
Chemotherapy is one of the most conventional therapeutic strategies for the treatment of human cancers. Conventional chemotherapy drugs include adriamycin (ADM), cisplatin and 5-fluorouracil. ADM is commonly used in the treatment of a wide range of cancers, including hematological malignancies, many types of carcinomas, and soft tissue sarcomas. Although the mechanism is not yet fully understood, ADM is generally believed to kill cancer cells by DNA intercalation, which eventually leads to cell death. Despite these merits, most serious adverse effect of ADM is life-threatening heart damage. It is therefore crucial to explore novel approaches to reduce drug dosage, minimize side effects and enhance therapeutic efficacy to promote the application of ADM in breast cancer chemotherapy. In this study, our aim was to investigate the combined effect of BPD-MA-mediated PDT plus ADM on 4T1 breast carcinoma cells in vivo and the underlying mechanism.

\section{Materials and methods Cell lines}

The 4T1 breast carcinoma cell line was cultured in RPMI-1640 (Invitrogen, Carlsbad, CA, USA) supplemented with 10\% heatinactivated fetal bovine serum (Gibco BRL, Grand Island, NY, USA), 100 units/mL penicillin (Sigma, St Louis, MO, USA), and $100 \mu \mathrm{g} / \mathrm{mL}$ streptomycin (Sigma, St Louis, MO, USA) and maintained in $5 \% \mathrm{CO}_{2}$ at $37{ }^{\circ} \mathrm{C}$.

\section{Animal experiments}

Five- to six-week-old female normal BALB/c mice were purchased from the Institute of Laboratory Animal Science in the Chinese Academy of Medical Sciences. 4T1 cells were collected and prepared as a $0.2-\mathrm{mL}$ cell suspension containing $8 \times 10^{6}$ cells; the cells were inoculated subcutaneously (sc) into the right flank of normal BALB/c mice using a sterile 22-gauge needle after alcohol preparation of the skin and manual restraint. Tumor sizes were measured using a caliper, and tumor volume was calculated using the formula $\left(\right.$ length $\times$ width $\left.^{2}\right) / 2$.

Treatment was started when the tumor mass reached approximately $100-150 \mathrm{~mm}^{3}$ in volume (d 4). On d 4, the mice were treated with ADM $(5 \mathrm{mg} / \mathrm{kg})$ via tail vein injection. On $\mathrm{d} 5$, the mice were treated with BPD-MA $(1 \mathrm{mg} / \mathrm{kg})$ via tail vein injection and were irradiated with a single dose of laser light (690 nm, energy density of $\left.120 \mathrm{~J} / \mathrm{cm}^{2}\right) 24 \mathrm{~h}$ later. On the indicated day, all tumors were collected to measure the tumor weights. All animal experiments were performed according to a protocol approved by the National Guidelines for Use and Care of Animals.

\section{Western blot}

To determine the levels of protein expression, tumor tissues were lysed in RIPA lysis buffer $[50 \mathrm{mmol} / \mathrm{L}$ Tris- $\mathrm{HCl}(\mathrm{pH} 8.0)$, $150 \mathrm{mmol} / \mathrm{L} \mathrm{NaCl}, 0.1 \%$ SDS, 1\% NP-40, 0.25\% sodium deoxycholate, and $1 \mathrm{mmol} / \mathrm{L}$ EDTA] containing a cocktail of freshly added protease inhibitors (Roche, USA) for $30 \mathrm{~min}$ on ice and subsequently centrifuged at $13000 \mathrm{r} / \mathrm{min}$ for $10 \mathrm{~min}$. Total protein concentration of the whole-cell extracts was measured using Bradford reagent (Bio-Rad, Hercules, CA, USA). The proteins were resolved by SDS-PAGE (Bio-Rad, Hercules, CA, USA). After electrophoresis, the proteins were electrotransferred onto polyvinylidene fluoride membranes (Millipore, Bedford, MA, USA), blocked with 5\% skim milk, and probed with the Bcl-2, Bax or $\beta$-actin primary antibody (Upstate Biotechnology, Lake Placid, NY, USA) that had been diluted in PBS/BSA followed by horseradish peroxidase (HRP)-conjugated secondary antibody (Sigma, St Louis, MO, USA). Binding was detected by enhanced chemiluminescence (Millipore, Bedford, MA, USA).

\section{Apoptosis assay}

Single-cell suspensions were made from tumor tissues, washed twice in PBS and incubated with $10 \mu \mathrm{L}(20 \mu \mathrm{g} / \mathrm{mL})$ of Annexin V-FITC according to the manufacturer's instructions. The cells were incubated with $5 \mu \mathrm{L}(50 \mu \mathrm{g} / \mathrm{mL})$ of propidium iodide for 2 min on ice and were analyzed with a FACScan flow cytometer (Beckman Counter Epics XL, USA). This assay discriminates between intact cells $\left(\mathrm{FITC}^{-} / \mathrm{PI}^{-}\right)$, early apoptotic cells $\left(\mathrm{FITC}^{+} / \mathrm{PI}^{-}\right)$, late apoptotic cells $\left(\mathrm{FITC}^{+} / \mathrm{PI}^{+}\right)$and necrotic cells $\left(\mathrm{FITC}^{-} / \mathrm{PI}^{+}\right)$.

\section{Immunohistochemistry}

Tumors were excised, fixed in $4 \%$ paraformaldehyde, and processed for paraffin embedding. Tumor tissue sections were immunostained using CD34 ${ }^{[7]}$ (Santa Cruz Biotechnology, USA) in addition to biotinylated secondary antibodies and horseradish peroxidase-conjugated anti-biotin from the ABC-Peroxidase Kit (Vector Laboratories, Burlingame, CA, USA) according to the manufacturer's instructions. Tumors were viewed under a light microscope (Olympus-IX70, Tokyo, Japan).

\section{Statistical analysis}

Results are expressed as the mean $\pm S D$, and two-tailed $t$-tests were used to evaluate the inter-group differences with Prism 5.0 software (GraphPad, USA). The statistical significance was set at $P<0.05$, and $P<0.01$ indicates a highly significant difference.

\section{Results}

PDT plus ADM suppresses in vivo 4T1 breast carcinoma growth

To determine whether PDT plus ADM inhibits 4T1 breast carcinoma growth in vivo, we carried out tumor growth experiments using 4T1 xenografted tumors in a normal BALB/c mouse model. As shown in Figure 1, tumor growth was more significantly retarded in the PDT plus ADM group compared with the PDT or ADM single treated group $(P<0.01)$, indicating that PDT plus ADM combination treatment remarkably suppressed tumor growth in vivo. To assess PDT plus ADMmediated prolongation of survival time of mice bearing 4T1 breast carcinoma cells, the mouse survival rate was investigated. As shown in Figure 2, the survival rate was significantly increased in the PDT plus ADM group compared with the PDT- or ADM-single treated group $(P<0.01)$. These results 


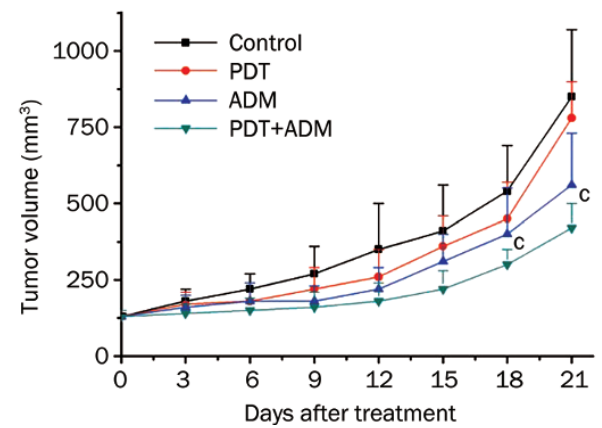

Figure 1. PDT plus ADM inhibits the 4T1 tumor growth in normal BALB/ c mice. 4T1 cells were collected and inoculated into female normal $\mathrm{BALB} / \mathrm{c}$ mice subcutaneously (sc) on one side of flank, $8 \times 10^{6}$ cells $/ 0.2$ $\mathrm{mL}$. The tumor sizes were measured using a caliper, tumor volume was calculated using the formula: (length $\times$ width $\left.^{2}\right) / 2$. On Day 21 , all tumors were collected to measure the tumor weights. Mean \pm SD. $n=6 .{ }^{c} P<0.01$.

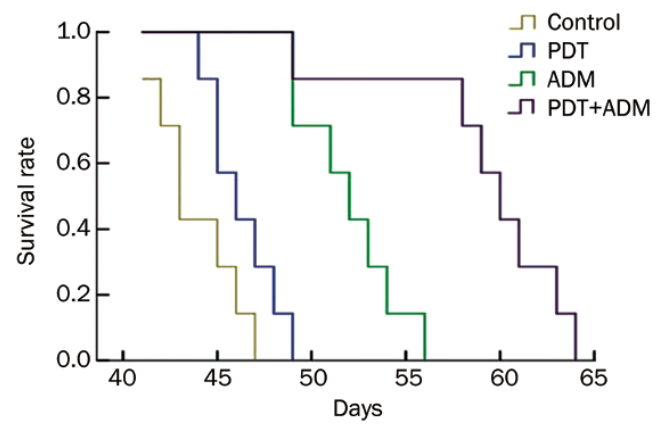

Figure 2. Effect of PDT plus ADM on the life span of $4 T 1$ transplantable murine breast carcinoma in normal BALB/c mice. Survival time of mice treated with PDT plus ADM was significantly longer than that of nontreated, single PDT and ADM-treated mice. Mean \pm SD. $n=6$. ${ }^{c} P<0.01$.

indicated that the combination of PDT and ADM prolongs the survival time of $4 \mathrm{~T} 1$ breast carcinoma-bearing mice.

PDT plus ADM enhances apoptosis in $4 \mathrm{~T} 1$ breast carcinoma cells We determined that PDT plus ADM enhanced apoptosis in 4T1 breast carcinoma cells (Figure 3). To examine whether PDT plus ADM combination treatment elicited enhanced apoptosis in breast carcinoma tissues, single-cell suspensions produced from different treatments of $4 \mathrm{~T} 1$ breast carcinoma tissues were stained with Annexin V (early apoptotic marker) and PI (late apoptotic marker). Apoptosis of $4 \mathrm{~T} 1$ breast carcinoma cells was subsequently analyzed by flow cytometry. As shown in Figure 4A, the combination of Ad-PDT with ADM more efficiently induced $4 \mathrm{~T} 1$ breast carcinoma apoptosis than PDT or ADM single. To further address the underlying molecular mechanism by which PDT plus ADM combination treatment results in an enhanced antitumor effect, the expression of apoptosis-related proteins, such as Bax and Bcl-2, was determined by Western blot analysis in differentially treated 4T1 xenografted tumor tissues. As shown in Figure 4B, expression of Bax in the PDT, ADM, and PDT plus ADM groups signifi-
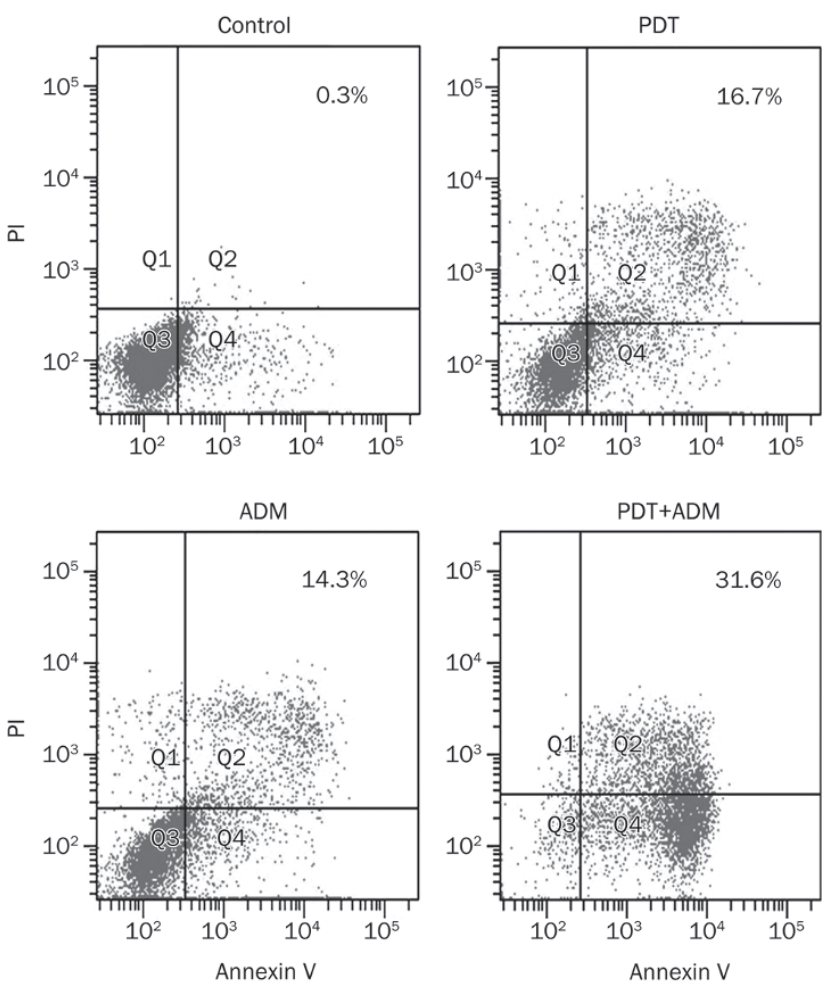

Figure 3. PDT plus ADM enhances apoptosis in 4T1 breast carcinoma cells. 4T1 breast carcinoma cells were treated with PDT $(0.5 \mu \mathrm{g} / \mathrm{mL}$, $\left.2 \mathrm{~J} / \mathrm{cm}^{2}\right), A D M(1 \mu \mathrm{g} / \mathrm{mL})$, PDT plus ADM, then the apoptotic cells were assessed by flow cytometry after $24 \mathrm{~h}$, respectively.

cantly increased, whereas the expression of Bcl-2 decreased in comparison with the control group. These results indicate that PDT plus ADM remarkably suppressed 4T1 breast carcinoma growth and induced apoptosis, which was closely associated with cooperative regulation of the intrinsic apoptotic pathway.

\section{PDT plus ADM reduces tumor vessel CD34 expression and MVD}

The positive expression of CD34 was mainly represented as brownish yellow or brownish granules in vascular endothelial cells. In all collected $4 \mathrm{~T} 1$ xenografted tumors, CD34 expression in tumor vascular endothelial cells in the PDT plus ADM combination treatment group was weaker than in the PDT- or ADM-single treated groups (Figure 5A), which indicated that PDT plus ADM additively or synergistically downregulates CD34 expression in 4T1 xenografted tumor vessels. Furthermore, the MVD (Figure 5B) in the PDT plus ADM group was significantly less than that in the PDT- or ADM-single groups. This result indicated that PDT plus ADM has a combined effect on the reduction of MVD in 4T1 xenografted tumors, which may be another important mechanism responsible for the PDT plus ADM-mediated in vivo combined growth inhibition of $4 \mathrm{~T} 1$ xenografted tumors in a normal BALB/c mouse model.

\section{Discussion}

At present, PDT is being tested in the oncology clinic to treat 

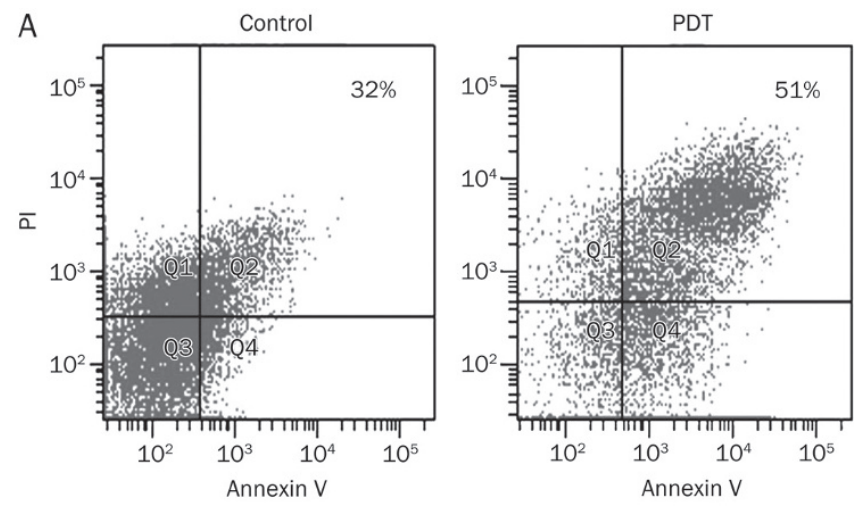

B

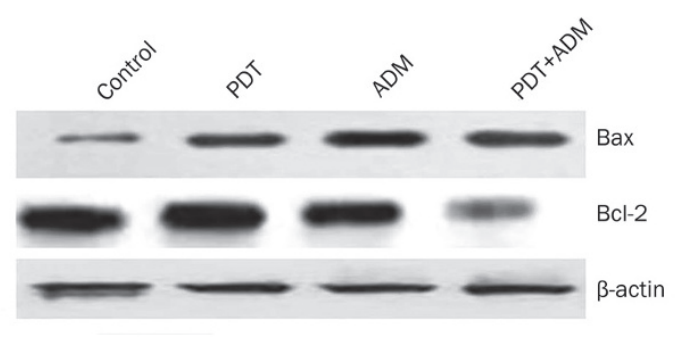

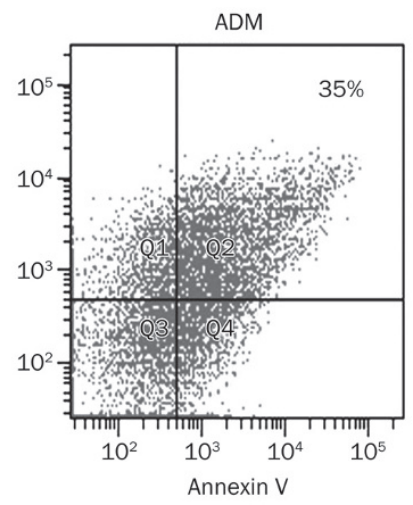
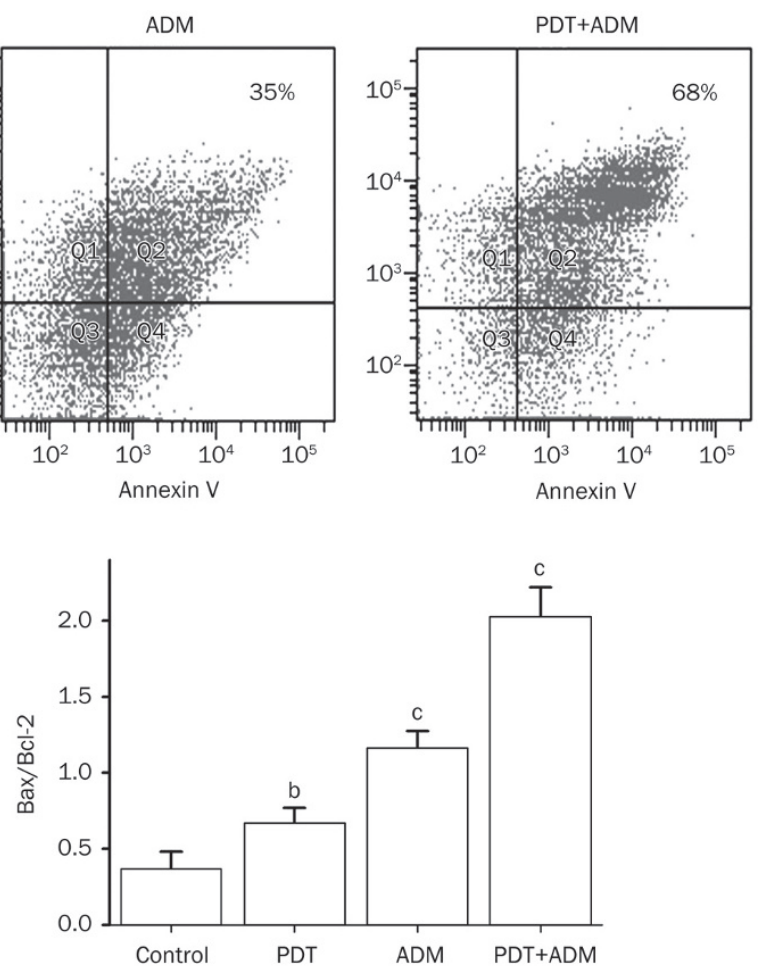

Figure 4. PDT plus ADM enhances apoptosis by cooperatively regulating extrinsic apoptotic pathways. Normal BALB/c mice bearing 4 T1 breast carcinoma were treated with PDT, ADM, PDT plus ADM, then the apoptotic cells and expression of apoptosis-related proteins in 4T1 tumor tissues were assessed by flow cytometry and Western blot analysis after $21 \mathrm{~d}$, respectively. (A) PDT plus ADM enhances apoptosis in 4T1 breast carcinoma cells. The apoptotic cells were analyzed using Annexin V/PI double staining by flow cytometry. The Annexin V single-positive cells (early apoptotic cells) in the total cell population represented apoptotic cells. PDT plus ADM enhanced apoptosis in 4T1 tumor cells $\left({ }^{b} P<0.05\right.$, compared with PDT and ADM group, one-way repeated measures ANOVA and multiple comparisons, $n=6$ replicates per condition). (B) PDT plus ADM cooperatively regulates extrinsic apoptotic pathways. Total tumor tissue lysates of treated and untreated 4T1 tumors were analyzed by immunoblotting with a panel of antibodies specific for Bcl-2 and Bax. The quantities of protein expression were normalized to the internal control $\beta$-actin measured in the same samples. PDT plus ADM combination treatment elicited an additive effect on the altered expression of apoptosis-related proteins such as Bcl-2 and Bax. Mean \pm SD. $n=3 .{ }^{\mathrm{b}} P<0.05,{ }^{\mathrm{c}} P<0.01$.

cancers of the head and neck, brain, lung, pancreas, intraperitoneal cavity, breast, prostate and $\operatorname{skin}^{[8]}$. The combination of various therapeutic modalities with non-overlapping toxicities is a commonly used strategy to improve the therapeutic index of treatments in modern oncology. However, the combined effect of PDT and ADM, an attractive chemotherapy drug, on breast cancer is still unknown. Therefore, we designed this study to address the therapeutic effect and potential mechanism of PDT plus chemotherapy by combining PDT with $\mathrm{ADM}$ and treating breast carcinoma cells in a normal BALB/c mouse model. We first assessed the inhibition of $4 \mathrm{~T} 1$ breast carcinoma growth by PDT plus ADM. We found that PDT plus ADM significantly inhibited $4 \mathrm{~T} 1$ breast carcinoma growth in a normal BALB/c mouse model. Additionally, the combination of PDT plus ADM prolonged the survival time of 4T1 breast carcinoma-bearing mice. To further address the underlying mechanism involved in the enhanced PDT plus ADMinduced antitumor activity, the expression of apoptosis-related proteins Bcl-2 and Bax in different $4 \mathrm{~T} 1$ breast carcinoma treatment groups was assessed by Western blot analysis. Tumor development requires a combination of defects that allows nascent neoplastic cells to become self-sufficient for cell proliferation and insensitive to signals that normally restrain cell growth. The evasion of apoptosis has proven to be critical for the development and sustained growth of cancer. The Bcl-2 protein family is known to be a key regulator of apoptosis and an important determinant of cell fate ${ }^{[9]}$. Mitochondria participate in apoptosis through a range of mechanisms that vary between vertebrates and invertebrates. In vertebrates, they release intermembrane space proteins, such as cytochrome $c$, to promote caspase activation in the cytosol. This process is the result of the loss of integrity of the outer mitochondrial membrane caused by proapoptotic members of the Bcl-2 family ${ }^{[9]}$. Moreover, Pluta et a $l^{[10]}$ showed that decreased Bax protein expression in breast cancer cells may suggest a potential means of apoptosis avoidance in tumor cells. Correlations among Bax protein, $\mathrm{p} 53$ and caspase-3 expression are probably associated with an active apoptotic mechanism in breast cancer cells expressing the Bax protein. Wu et al ${ }^{[11]}$ also reported that Bax activation is not essential for mitochondrial outer membrane permeabilization but essential for Drp1-mediated mitochondrial fission during the apoptosis caused by Photofrin- 
A
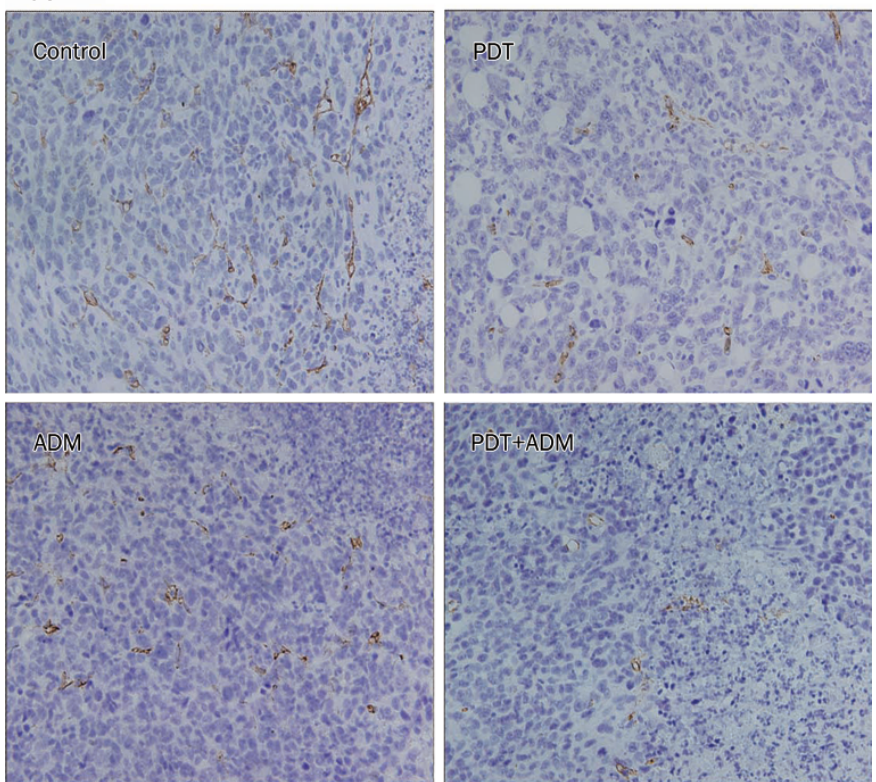

B

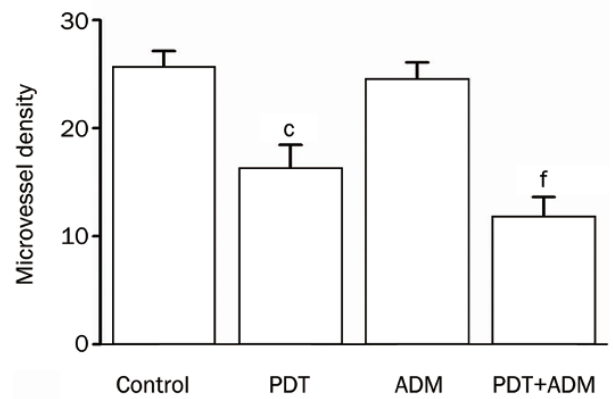

Figure 5. Combined inhibition of tumor angiogenesis in vivo. (A) Immunohistochemical detection for CD34 expression in 4T1 breast carcinoma sc xenografted tumors. Representative pictures for different treatment groups are shown. The positive expression of CD34 was mainly represented as brownish yellow or brownish granules in tumor vascular endothelial cells. (B) The tumor MVD in different treatment groups. PDT plus ADM combination treatment synergistically inhibited in vivo angiogenesis of $4 \mathrm{~T} 1$ breast carcinoma sc xenografted tumors $\left({ }^{c} P<0.01\right.$ vs control group, one-way repeated measures ANOVA and multiple comparisons, $n=6$ mice per condition, $n=5$ observations per representative section). ${ }^{f} P<0.01$ vs $\mathrm{PDT}$ or $\mathrm{ADM}$ single treatment group.

PDT. The ratio of anti- to pro-apoptotic molecules constitutes a rheostat that sets the threshold of susceptibility to apoptosis for the intrinsic apoptotic pathway, which promotes pore formation in the mitochondrial outer membrane, leading to the loss of mitochondrial integrity and the release of cytochrome $c$ into the cytosol ${ }^{[12-14]}$. Western blot results showed that PDT plus ADM combination treatment elicited an additive effect on the altered expression of apoptosis-related proteins, leading to the cooperative activation of the intrinsic apoptotic pathway, which may closely account for the PDT plus ADM-mediated growth inhibition and apoptosis in 4T1 breast carcinoma cells.

Angiogenesis has been a common prognostic indicator for breast carcinoma in the last decade. Indeed, previous studies have shown that higher intratumoral microvessel density is statistically correlated with a greater incidence of metastasis and that intratumoral microvessel density is an independent prognostic indicator for overall and relapse-free survival in early-stage invasive breast carcinomas ${ }^{[15,16]}$. In addition to directly killing cancer cells, PDT appears to shrink or destroy tumors in other ways. The photosensitizer can damage blood vessels in the tumor, thereby preventing the cancer from receiving necessary nutrients ${ }^{[17-19]}$. Gomer et $\mathrm{al}^{[20]}$ showed that bovine endothelial cells were significantly more sensitive to PDT with porfimer sodium than smooth muscle cells or fibroblasts from the same species. To explore the combined effect of PDT plus ADM on tumor angiogenesis in vivo, the MVD in 4T1 xenograft tumour tissues was determined by CD34 immunohistochemistry analysis. We found that PDT plus ADM downregulated CD34 expression and suppressed angiogenesis in $4 \mathrm{~T} 1$ breast carcinoma, which may be another important mechanism involved in the PDT plus ADM-mediated in vivo combined growth inhibition of 4T1 xenografted tumors in normal BALB/c mice.

Taken together, the PDT plus ADM combined treatment resulted in in vivo combined growth inhibition and enhanced apoptosis, as well as imparting an additive effect on Bax upregulation and Bcl-2 downregulation in $4 \mathrm{~T} 1$ breast carcinomas. This treatment also reduced CD34 expression and MVD in 4T1 xenografted tumors. Most importantly, the enhanced in vivo antitumor effect elicited by PDT plus ADM was closely associated with an increase in Bax expression and a decrease in the ratio of anti- to pro-apoptotic molecules of Bcl-2 by cooperatively regulating intrinsic apoptotic pathways and the in vivo combined inhibition of tumor angiogenesis. Therefore, our results indicate that BPD-MA-mediated PDT plus ADM is a potential combined treatment strategy against breast carcinoma.

\section{Acknowledgements}

This work was supported by the Natural Science Foundation of Tianjin [No 10JCYBJC11500].

\section{Author contribution}

Zhong-sheng TONG designed research; Pei-tian MIAO performed research; Xiao-dong LIU contributed new reagents or analytic tools; Ting-ting LIU analyzed data; Yong-sheng JIA wrote the paper.

\section{References}

1 Buchholz TA. Introduction: locally advanced breast cancer. Semin Radiat Oncol 2009; 19: 193-4.

2 Gould J, Fitzgerald B, Fergus K, Clemons M, Baig F. Why women delay seeking assistance for locally advanced breast cancer. Can Oncol Nurs J 2010; 20: 23-9.

3 Triesscheijn M, Baas P, Schellens JH, Stewart FA. Photodynamic therapy in oncology. Oncologist 2006; 11: 1034-44.

4 Agostinis P, Berg K, Cengel KA, Foster TH, Girotti AW, Gollnick SO, et al. Photodynamic therapy of cancer: an update. CA Cancer J Clin 2011; 61: 250-81. 
5 Wyss P, Schwarz V, Dobler-Girdziunaite D, Hornung R, Walt H, Degen A, et al. Photodynamic therapy of locoregional breast cancer recurrences using a chlorin-type photosensitizer. Int J Cancer 2001; 93: $720-4$.

6 Aveline B, Hasan T, Redmond RW. Photophysical and photosensitizing properties of benzoporphyrin derivative monoacid ring A (BPD-MA). Photochem Photobiol 1994; 59: 328-35.

7 Weidner N. Measuring intratumoral microvessel density. Methods Enzymol 2008; 444: 305-23.

8 Dolmans DE, Fukumura D, Jain RK. Photodynamic therapy for cancer. Nat Rev Cancer 2003; 3: 380-7.

9 Kelly PN, Strasser A. The role of Bcl-2 and its pro-survival relatives in tumourigenesis and cancer therapy. Cell Death Differ 2011; 18 : 1414-24.

10 Pluta P, Smolewski P, Pluta A, Cebula-Obrzut B, Wierzbowska A, Nejc $D$, et al. Significance of bax expression in breast cancer patients. Pol Przegl Chir 2011; 83: 549-53.

11 Wu S, Zhou F, Zhang Z, Xing D. Bax is essential for Drp1-mediated mitochondrial fission but not for mitochondrial outer membrane permeabilization caused by photodynamic therapy. J Cell Physiol 2011; 226: 530-41.

12 Martinou JC, Youle RJ. Mitochondria in apoptosis: Bcl-2 family members and mitochondrial dynamics. Dev Cell 2011; 21: 92-101.

13 Fletcher J, Meusburger S, Hawkins CJ, Riglar DT, Lee EF, Fairlie WD, et al. Apoptosis is triggered when prosurvival $\mathrm{Bcl}-2$ proteins cannot restrain Bax. Proc Natl Acad Sci U S A 2008; 105: 18081-7.

14 Eissing T, Waldherr S, Allgower F, Scheurich P, Bullinger E. Response to bistability in apoptosis: roles of bax, bcl-2, and mitochondrial permeability transition pores. Biophys J 2007; 92: 3332-4.

15 Vartanian RK, Weidner N. Correlation of intratumoral endothelial cell proliferation with microvessel density (tumor angiogenesis) and tumor cell proliferation in breast carcinoma. Am J Pathol 1994; 144: 1188-94.

16 Weidner N. Current pathologic methods for measuring intratumoral microvessel density within breast carcinoma and other solid tumors. Breast Cancer Res Treat 1995; 36: 169-80.

17 Chen B, Pogue BW, Luna JM, Hardman RL, Hoopes PJ, Hasan T. Tumor vascular permeabilization by vascular-targeting photosensitization: effects, mechanism, and therapeutic implications. Clin Cancer Res 2006; 12: 917-23.

18 Bhuvaneswari R, Gan YY, Soo KC, Olivo M. The effect of photodynamic therapy on tumor angiogenesis. Cell Mol Life Sci 2009; 66: 2275-83.

19 Lisnjak IO, Kutsenok VV, Polyschuk LZ, Gorobets OB, Gamaleia NF. Effect of photodynamic therapy on tumor angiogenesis and metastasis in mice bearing Lewis lung carcinoma. Exp Oncol 2005; 27: 333-5.

20 Gomer CJ, Rucker N, Murphree AL. Differential cell photosensitivity following porphyrin photodynamic therapy. Cancer Res 1988; 48: 4539-42. 